\title{
Pressure gradient measurement in the coronary artery using phase contrast (PC)-MRI: initial patient results towards noninvasive quantification of fractional flow reserve
}

\author{
Zixin Deng ${ }^{1,2^{*}}$, Sang Eun Lee ${ }^{5}$, Zhaoyang Fan ${ }^{1}$, Christopher T Nguyen ${ }^{1}$, Qi Yang ${ }^{1}$, Xiaoming Bi ${ }^{3}$, \\ Byoung Wook Choi ${ }^{4}$, Daniel S Berman ${ }^{1}$, Hyuk-Jae Chang ${ }^{5}$, Debiao Li, ${ }^{1,2}$
}

From 19th Annual SCMR Scientific Sessions

Los Angeles, CA, USA. 27-30 January 2016

\section{Background}

Fractional flow reserve (FFR) is an invasive procedure evaluating the functional significance of an intermediate coronary stenosis in patients with coronary artery disease (CAD) [1]. Quantification of pressure gradient $(\Delta \mathrm{P})$ across a particular stenosis is the key to the determination of FFR. Noninvasive $\Delta \mathrm{P}$ measurement $\left(\Delta \mathrm{P}_{\mathrm{MR}}\right)$ using phase-contrast (PC)-MRI in conjunction with NavierStokes (NS) equations has been attempted in various vessels [2-4]. Our previous work has shown the feasibility of deriving $\Delta \mathrm{P}_{\mathrm{MR}}$ at various vessel diameters in a phantom (fig.1a) and excellent correlation between $\Delta \mathrm{P}_{\mathrm{MR}}$ and $\Delta \mathrm{P}$ measured via a pressure-transducer (fig.1b). This study aimed to investigate the feasibility of deriving $\Delta \mathrm{P}_{\mathrm{MR}}$ in healthy and diseased coronary arteries.

\section{Methods}

Coronary PC-MRI acquisitions were ECG triggered (mid-diastole) and navigator gated (end-expiration) [5]. Fat-suppression pre-pulses were applied prior to the acquisitions to avoid chemical shift effects and increase vessel contrast [6]. Contiguous slices (4-9) were consecutively collected across the proximal coronary segment (healthy controls) or stenotic lesion (patients). Imaging parameters were: VENC $=35-65 \mathrm{~cm} / \mathrm{s}$ in all 3 directions, $\mathrm{FA}=15^{\circ}$, cardiac phase $=2(\sim 70 \mathrm{~ms} /$ phase $)$, in-plane resolution $=0.5-0.6 \times 0.5-0.6 \mathrm{~mm}^{2}$, slice thickness $=3.2 \mathrm{~mm}$ and $\mathrm{TA}=2-4 \mathrm{~min} / \mathrm{slice}$ at $3 \mathrm{~T}$. Eddy-current correction was done offline followed by NS calculations [7].

${ }^{1}$ Cedars Sinai Medical Center, Los Angeles, CA, USA

Full list of author information is available at the end of the article
Protocol was performed on 11 healthy controls and 6 patients (one with known invasive FFR). Patient inclusion criteria: known/suspected CAD, $\geq 1$ coronary lesion (proximal stenosis $\geq 30 \%$ ) detected by CTA and/or invasive coronary angiography (ICA).

\section{Results}

A significant $(p<0.001)$ increase in $\Delta \mathrm{P}_{\mathrm{MR}}$ was seen in the patient group $(6.40 \pm 4.43 \mathrm{mmHg})$ vs. healthy controls $(0.62 \pm 0.49 \mathrm{mmHg}$ ) (figre $2 a)$. CTA/ICA reports in $5 / 6$ patients showed a range of stenoses of $30-50 \%$ (proximal left anterior descending coronary artery ( $p \mathrm{LAD})$ ), but not significant enough to perform invasive FFR. ICA/FFR was performed in $1 / 6$ patients (diffused, 50\% lumen narrowing at $p \mathrm{LAD}$, fig $2 b-c$ ) with FFR $=0.56$, suggesting a functionally significant lesion. The same patient showed a $\Delta \mathrm{P}_{\mathrm{MR}}$ of $\sim 14 \mathrm{mmHg}$, likewise suggesting a functionally significant lesion (relatively high pressure drop).

\section{Conclusions}

Preliminary results suggest that noninvasive quantification of $\Delta \mathrm{P}_{\mathrm{MR}}$ in both healthy and diseased coronary arteries is feasible. The patient with low FFR (high pressure drop), corroborating the $\Delta \mathrm{P}_{\mathrm{MR}}$ results, showed the feasibility of $\Delta \mathrm{P}_{\mathrm{MR}}$ in differentiating between a functionally significant and a non-significant lesion within the patient group. More patient studies with invasive FFR comparison are underway to further validate the approach. In addition, technical improvements in terms of spatial, temporal resolutions and reduction of noise are also being developed to further improve accuracy. 


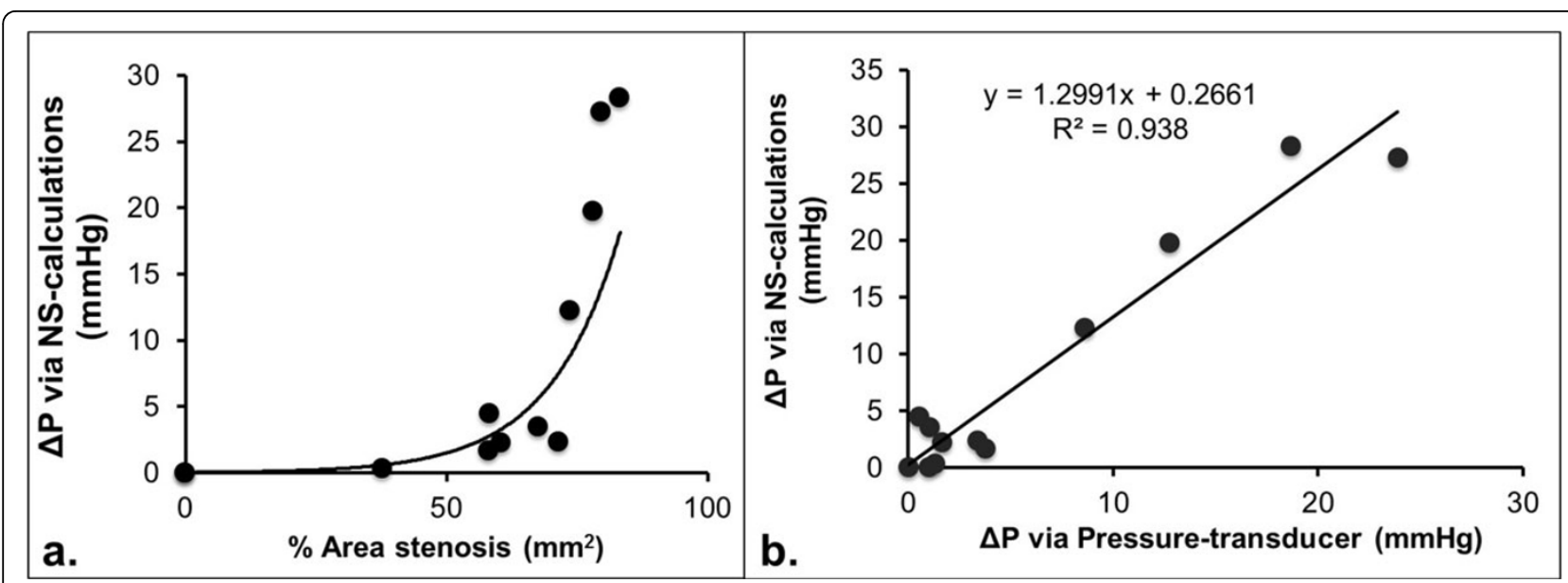

Figure $\mathbf{1}$ a) $\%$ area stenosis versus $\mathbf{P C}-\mathbf{M R I}$ derived $\Delta \mathbf{P}$ measurement. An exponential increase in $\Delta \mathrm{P}$ was observed as $\%$ area stenosis increases. b) Pressure measurement comparison between $\Delta P$ calculated via NS-equations and $\Delta$ measured using pressure transducer. Excellent correlation $\left(R^{2}=0.938\right)$ was observed between the two techniques, validating the feasibility of PC-MRI derived $\triangle P$.

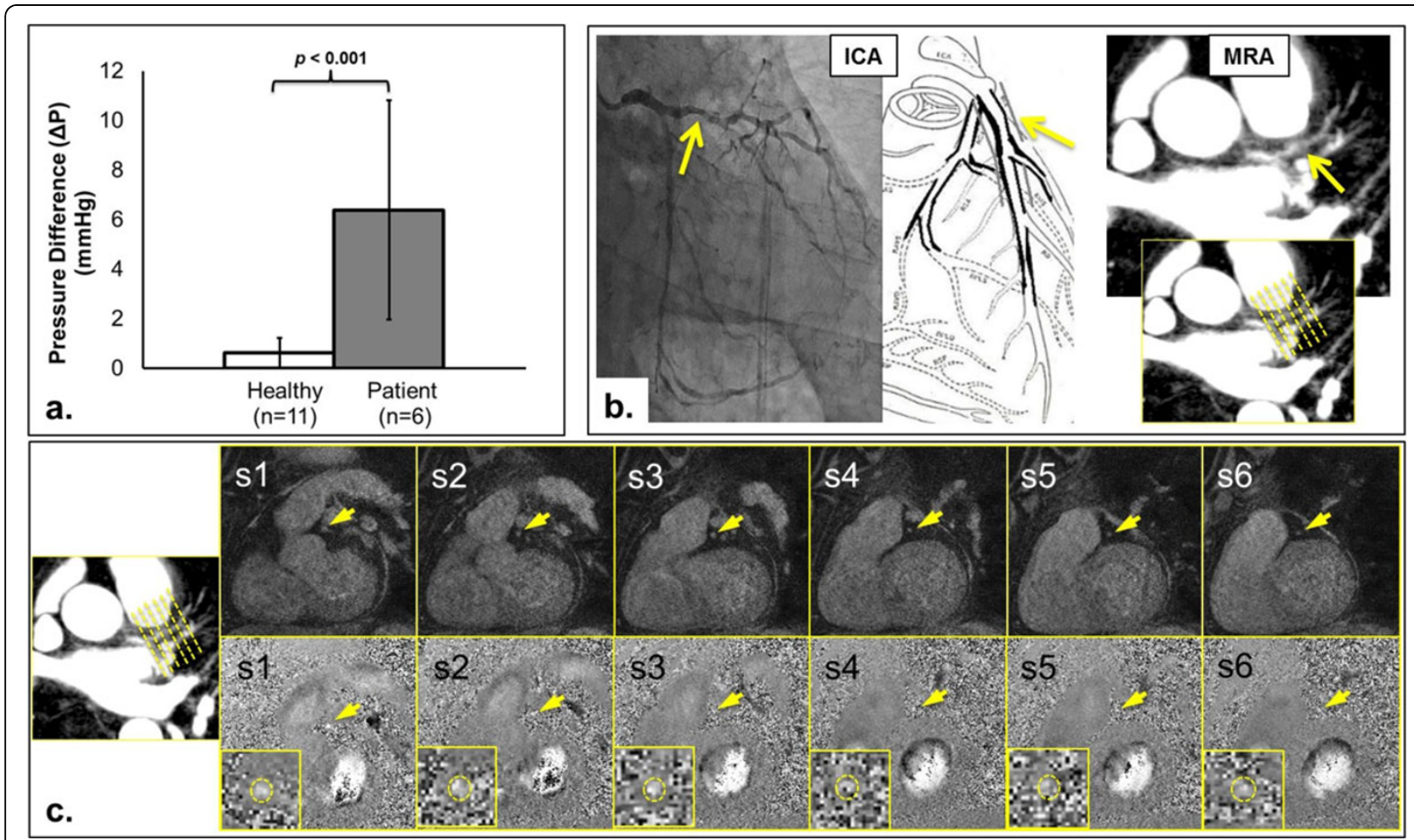

Figure 2 a) The mean \pm std $\Delta \mathrm{P}$ value for health controls and patients were $0.6249 \pm 0.4884 \mathrm{mmHg}$ and $6.3960 \pm 4.4302 \mathrm{mmHg}$, respectively. The high standard deviation in the patient group is due to the range of the stenotic level among the patient group. b) Patient example (Patient A). Invasive coronary angiography (ICA) and magnetic resonance angiography (MRA). c) Patient A, six cross-sectional slices obtained from PC-MRI over the stenotic lesion at the proximal left anterior descending artery. Top row: flow compensated, bottom row: phase contrast (in the z-direction). 


\section{Authors' details}

${ }^{1}$ Cedars Sinai Medical Center, Los Angeles, CA, USA. ${ }^{2}$ Bioengineering, University of California, Los Angeles, Los Angeles, CA, USA. ${ }^{3}$ R\&D, Siemens Healthcare, Los Angeles, CA, USA. ${ }^{4}$ Radiology, Severance Hospital, Yonsei University College of Medicine, Seoul, Korea (the Republic of). ${ }^{5}$ Cardiology, Severance Cardiovascular Hospital, Yonsei University College of Medicine, Seoul, Korea (the Republic of).

Published: 27 January 2016

\section{References}

1. Pijls, et al: NEJM 1996

2. Bock, et al: MRM 2011

3. Lum, et al: RY 2007.

4. Bley, et al: RY 2011.

5. Deng, et al: ISMRM 2014.

6. Middione, et al: MRM 2013.

7. Yang, et al: MRM 1996.

doi:10.1186/1532-429X-18-S1-P218

Cite this article as: Deng et al:. Pressure gradient measurement in the coronary artery using phase contrast (PC)-MRI: initial patient results towards noninvasive quantification of fractional flow reserve. Journal of Cardiovascular Magnetic Resonance 2016 18(Suppl 1):P218.

\section{Submit your next manuscript to BioMed Central} and take full advantage of:

- Convenient online submission

- Thorough peer review

- No space constraints or color figure charges

- Immediate publication on acceptance

- Inclusion in PubMed, CAS, Scopus and Google Scholar

- Research which is freely available for redistribution

Submit your manuscript at www.biomedcentral.com/submit 